\title{
Some Common Fixed Point Theorems in Menger Space
}

\author{
Sanjay Kumar', Asha Rani ${ }^{2,3}$ \\ ${ }^{1}$ Departement of Mathematics, Deenbandhu Chhotu Ram University of Science and Technology, Sonepat, India \\ ${ }^{2}$ Department of Applied Sciences, B. M. Institute of Engineering and Technology, Sonepat, India \\ ${ }^{3}$ DCRUST, Sonepat, India \\ Email: sanjaymudgal2004@yahoo.com, asha.dahiya27@gmail.com
}

Received November 19, 2011; revised February 6, 2012; accepted February 14, 2012

\begin{abstract}
This paper consists four sections. First section is central to the text. In second section, we generalize the results of Kohli and Vashistha [1] for pairs of mappings using weakly compatible maps. Third section deals the results for pair of weakly compatible maps along with property (E.A.) using different types of control functions, which generalize the results of Kohli and Vashistha [1] and Kubiaczyk and Sharma [2]. Fourth section is concerned with results for occasionally weakly compatible maps and generalizes, extends and unifies several well known comparable results in literature.
\end{abstract}

Keywords: Weakly Compatible Maps; Occasionally Weakly Compatible Maps; Property (E.A.); Common Property (E.A.)

\section{Introduction}

In 1942 Menger [3] introduced the notion of a probabilistic metric space (PM-space) which is in fact, a generalization of metric space. The idea in probabilistic metric space is to associate a distribution function with a point pair, say $(p, q)$, denoted by $F(p, q, t)$ where $t>0$ and interpret this function as the probability that distance between $p$ and $q$ is less than $t$, whereas in the metric space the distance function is a single positive number. Sehgal [4] initiated the study of fixed points in probabilistic metric spaces. First, we recall that a real valued function defined on the set of real numbers is known as a distribution functions if it is non-decreasing, left continuous with $\inf f(x)=0$ and $\sup f(x)=1$. In what follows, $H(x)$ denotes the Heavy side function, a simple example of distribution function.

$$
H(x)=\left\{\begin{array}{lll}
0 & \text { if } & x \leq 0 \\
1 & \text { if } & x>0
\end{array}\right.
$$

Definition 1.1. A probabilistic metric space is a pair $(X, F)$, where $X$ is a non empty set and $F$ is a function defined $F: X \times X \rightarrow L$ (the set of all distribution functions) satisfying the following properties:

1) $F(x, y, 0)=0$,

2) $F(x, y, t)=H(t)$, iff $x=y$,

3) $F(x, y, t)=F(y, x, t)$, and

4) $F(x, y, s)=1$ and $F(y, z, t)=1$, then

$F(x, z, s+t)=1$,

for all $x, y, z \in X$ and $\mathrm{s}, t>0$.

For each $x$ and $y$ in $X$ and for each real number $t>0$,
$F(x, y, t)$ is to be thought of as the probability that the distance between $x$ and $y$ is less than $t$. Of course, a metric space $(X, d)$ induces a PM-space. Every metric space $(X, d)$ can be realized as a probabilistic metric space by taking $F: X \times X \rightarrow L$ : defined by $F(x, y, t)=H(t-d(x, y))$ for all $x, y$ in $X$.

Definition 1.2. A $t$-norm (in the sense of B. Schweizer and A. Sklar [5]) $\Delta$ is a 2-place function $\Delta:[0,1] \times[0,1] \rightarrow[0,1]$ satisfying the following:

1) $\Delta(0,0)=0$

2) $\Delta(0,1)=1$

3) $\Delta(a, b)=\Delta(b, a)$,

4) If $a \leq c$ and $b \leq d$ then $\Delta(a, b) \leq \Delta(c, d)$, and

5) $\Delta(\Delta(a, b), c)=\Delta(a, \Delta(b, c))$, for all $a, b, c \in[0,1]$.

Definition 1.3. A Menger PM-space is a triplet $(X, F$, $\Delta$ ) where $(X, F)$ is a PM-space and $\Delta$ is a $t$-norm with the following condition:

$$
F(x, z, s+t) \geq \Delta(F(x, y, s), F(y, z, t)) \text { for all } x, y, z
$$

$\in X$ and $s, t>0$.

This inequality is known as Menger's triangle inequality.

Definition 1.4. A sequence $\left\{x_{n}\right\}$ in $(X, F, \Delta)$ is said to

1) Converge to a point $x \in X$ if for every $\varepsilon>0$ and $\lambda>$ 0 , there exists a positive integer $N(\varepsilon, \lambda)$ such that $F\left(x_{n}, x, \varepsilon\right)>1-\lambda$ for all $n \geq N(\varepsilon, \lambda)$.

2) Be a Cauchy sequence if for every $\varepsilon>0$ and $\lambda>0$, there exists a positive integer $N(\varepsilon, \lambda)$ such that $F\left(x_{n}, x_{m}, \varepsilon\right)>1-\lambda$ for all $n, m \geq N(\varepsilon, \lambda)$.

A Menger space $(X, F, \Delta)$ is said to be complete if every Cauchy sequence in $X$ converges to a point in $X$. 
In 1972, the notion of contraction mappings on probabilistic metric spaces was first introduced by Sehgal et al. [4], that is, "every contraction mapping on a complete Menger space has a unique fixed point". Recently, Kohli and Vashistha [1] formulate the notion of $R$-weakly commuting mappings of type 1 ), $R$-weakly commuting mappings of type 2 ) and $R$-weakly commuting mappings of type 3 ) as follows:

Definition 1.5. A pair of self-mappings $(f, g)$ of a Menger probabilistic metric space $(X, F, \Delta)$ is said to be

1) Weakly commuting if $F(f g x, g f x, t) \geq F(f x, g x, t)$,

2) $R$-weakly commuting if there exists some $R>0$ such that $F(f g x, g f x, t) \geq F(f x, g x, t / R)$,

3) $R$-weakly commuting mappings of type 1$)$ if there exists some $R>0$ such that $F(g f x, f f x, t) \geq F(f x, g x, t / R)$,

4) $R$-weakly commuting mappings of type 2$)$ if there exists some $R>0$ such that

$F(f g x, g g x, t) \geq F(f x, g x, t / R)$,

5) $R$-weakly commuting mappings of type 3 ) if there exists some $R>0$ such that

$F(f f x, g g x, t) \geq F(f x, g x, t / R)$ for all $x \in X$ and $t>0$.

Moreover, such mappings commute at their coincidence points.

Now we state a Lemma which is useful for further study.

Lemma 1.1 [5]. Let $(X, F, \Delta)$ be a Menger space. If there exists $k \in(0,1)$ such that $F(x, y, k t) \geq F(x, y, t)$ for all $x, y \in X$ and $t>0$, then $x=y$.

\section{Weakly Compatible Maps}

In 1982, Sessa [6], weakened the concept of commutativity to weakly commuting mappings. Afterwards, Jungck [7] enlarged the concept of weakly commuting mappings by adding the notion of compatible mappings. In 1991, Mishra [8] introduced the notion of compatible mappings in the setting of probabilistic metric space.

\section{Definition 2.1 [8].}

Let $(X, F, \Delta)$ be a Menger space such that the t-norm $\Delta$ is continuous and $S, T$ be mappings from $X$ into itself. Then, $S$ and $T$ are said to be compatible if

$\lim _{n \rightarrow \infty} F\left(S T x_{n}, T S x_{n}, t\right)=1$ for all $t>0$, whenever $\left\{x_{n}\right\}$ is a sequence in $X$ such that $\lim _{n \rightarrow \infty} S x_{n}=\lim _{n \rightarrow \infty} T x_{n}=z$ for some $z \in X$.

In 1996, Jungck [9] introduce the notion of weakly compatible mappings as follows:

Definition 2.2. Two self mappings $S$ and $T$ are said to be weakly compatible if they commute at their coincide points, i.e., $T u=S u$ for some $u \in X$, then $T S u=S T u$.

Example 2.1. Let $X=[0,3]$ be equipped with the usual metric $d(x, y)=|x-y|$. Define $f, g:[0,3] \rightarrow[0,3]$ by

$$
f(x)=\left\{\begin{array}{lll}
x & \text { if } & x \in[0,1) \\
3 & \text { if } & x \in[1,3]
\end{array}\right.
$$

and

$$
g(x)=\left\{\begin{array}{lll}
3-x & \text { if } & x \in[0,1) \\
3 & \text { if } & x \in[1,3]
\end{array}\right.
$$

Then for any $x \in[1,3], x$ is a coincidence point and $f g x$ $=g f x$, showing that $f, g$ are weakly compatible maps on $[0$, 3].

Remark 2.1. Every weakly compatible map need not be compatible, see Example 2.2.

Kohli and Vashistha [1] proved the following result:

Theorem 2.1. Let $f$ and $g$ be $R$-weakly commuting self mappings of a Menger probabilistic metric space $(X, F$, $T$ ), where $T$ denotes a continuous t-norm, satisfying conditions

(a-I) $f(X) \subset g(X)$

(a-II) $F(f x, f y, t) \geq r F(g x, g y, t)$,

for all $x, y$ in $X$, where $r:[0,1] \rightarrow[0,1]$ is a continuous function such that $r(t)>t$ for each $0<t<1, r(0)=0$ and $r(1)=1$.

(a-III) If there exist sequences $\left\{x_{n}\right\}$ and $\left\{y_{n}\right\}$ in $X$ such that $x_{n} \rightarrow x, y_{n} \rightarrow y$ and $t>0$, then $F\left(x_{n}, y_{n}, t\right) \rightarrow F(x, y, t)$.

If one of the mappings $\mathrm{f}$ and $\mathrm{g}$ is continuous, then the mappings $f$ and $g$ have a unique common fixed point.

Remark 2.2. Recently, Mihet [10] proved that probabilistic version of Pant's theorem holds if some addition conditions 1) Every asymptotically regular sequence in $f(X)$ converges; 2) there exists $x_{0}$ in $X, x_{1} \in g^{-1}\left(f x_{0}\right)$ : $F\left(f x_{0}, f x_{1}, t\right)>0$, for all $\left.t>0 ; 3\right) x \neq y$ implies there exists $t>0: 0<F(x, y, t)<1)$ are imposed on the Theorems 4.7 and 4.8 of the paper [1]. The coditions 2) and 3) may be replaced by some stronger condition $F(x, y ; t)>0$ for all $x, y$ in $X$ and $t>0$.

Now we come to our main result.

Let $(X, F, \Delta)$ be a complete Menger probabilistic metric space and $\Delta(a, b)=\min (a, b)$ continuous $t$-norm. Let $A, B, S$ and $T$ be self-mappings of $X$ satisfying the following conditions:

$$
\begin{gathered}
A(X) \subset T(X) \text { and } B(X) \subset S(X), \\
F(A x, B y, t) \geq \varphi\{\min (F(S x, T y, t), F(S x, A x, t), \\
F(B y, T y, t), F(S x, B y, 2 t), F(A x, T y, t))\},
\end{gathered}
$$

for all $x, y \in X$, where $\varphi:[0,1] \rightarrow[0,1]$ is a continuous function such that $\varphi(s)>s$ for each $0<s<1$ with $F(x, y, t)>0$.

Then for any arbitrary point $x_{0} \in X$ by (2.1), we choose a point $x_{1} \in X$ such that $A x_{0}=T x_{1}$ and for this point $x_{1}$, there exists a point $x_{2} \in X$ such that $S x_{2}=B x_{1}$ and so on. Continuing in this way, we can construct a sequence $\left\{y_{n}\right\}$ in $X$ such that 


$$
\begin{gathered}
y_{2 n}=T x_{2 n+1}=A x_{2 n}, y_{2 n+1}=S x_{2 n+2}=B x_{2 n+1} \\
\text { for } n=0,1,2, \cdots
\end{gathered}
$$

To prove our main result, firstly we prove the following lemma:

Lemma 2.1. Let $A, B, S$ and $T$ be self-mappings of a
Menger probabilistic metric space $(X, F, \Delta)$, and $\Delta(a, b)=\min (a, b)$ continuous $t$-norm, satisfying the conditions (2.1) and (2.2). Then the sequence $\left\{y_{n}\right\}$ defined by (2.3) is a Cauchy sequence in $X$.

Proof. For $t>0$,

$$
\begin{aligned}
& F\left(y_{2 n}, y_{2 n+1}, t\right)=F\left(A x_{2 n}, B x_{2 n+1}, t\right) \\
& \geq \varphi\left\{\min \left(F\left(S x_{2 n}, T x_{2 n+1}, t\right), F\left(S x_{2 n}, A x_{2 n}, t\right), F\left(B x_{2 n+1}, T x_{2 n+1}, t\right), F\left(S x_{2 n}, B x_{2 n+1}, 2 t\right), F\left(A x_{2 n}, T x_{2 n+1}, t\right)\right)\right\} \\
& =\varphi\left\{\operatorname { m i n } \left(F\left(y_{2 n-1}, y_{2 n}, t\right),\left(F\left(y_{2 n-1}, y_{2 n}, t\right),\left(F\left(y_{2 n}, y_{2 n+1}, t\right),\left(F\left(y_{2 n-1}, y_{2 n+1}, 2 t\right),\left(F\left(y_{2 n}, y_{2 n}, t\right)\right)\right\}\right.\right.\right.\right. \\
& \geq \varphi\left\{\operatorname { m i n } \left(F\left(y_{2 n-1}, y_{2 n}, t\right),\left(F\left(y_{2 n-1}, y_{2 n}, t\right),\left(F\left(y_{2 n}, y_{2 n+1}, t\right), \Delta\left(\left(F\left(y_{2 n-1}, y_{2 n}, t\right)\right),\left(F\left(y_{2 n}, y_{2 n+1}, t\right)\right), 1\right)\right\}\right.\right.\right. \\
& >\left\{\begin{array}{l}
F\left(y_{2 n-1}, y_{2 n}, t\right) \text { if } F\left(y_{2 n-1}, y_{2 n}, t\right)<F\left(y_{2 n}, y_{2 n+1}, t\right) \\
F\left(y_{2 n}, y_{2 n+1}, t\right) \text { if } F\left(y_{2 n-1}, y_{2 n}, t\right) \geq F\left(y_{2 n}, y_{2 n+1}, t\right)
\end{array}\right.
\end{aligned}
$$

as $\varphi(s)>s$ for $0<s<1$. Thus

$\left\{F\left(y_{2 n}, y_{2 n+1}, t\right), n \geq 0\right\}$ is an increasing sequence of positive real numbers in $[0,1]$ and therefore tends to a limit $l \leq 1$. We assert that $l=1$. If not, $l<1$ which on letting $n \rightarrow \infty$ in (2.4), one gets $l \geq \varphi(l)>l$ a contradiction yielding thereby $l=1$. Therefore for every $n \in N$, using analogous arguments one can show that $\left\{F\left(y_{2 n+1}, y_{2 n+2}, t\right), n \geq 0\right\}$ is a sequence of positive real numbers in $[0,1]$ which tends to a limit $l=1$. Therefore for every $n \in N, F\left(y_{n}, y_{n+1}, t\right)>F\left(y_{n-1}, y_{n}, t\right)$ and $\lim _{n \rightarrow \infty} F\left(y_{n}, y_{n+1}, t\right)=1$. Now for any positive integer $p$, we obtain,

$F\left(y_{n}, y_{n+p}, t\right) \geq F\left(y_{n}, y_{n+1}, t / p\right) \Delta \cdots \Delta F\left(y_{n+p-1}, y_{n+p}, t / p\right)$

Since $\lim _{n \rightarrow \infty} F\left(y_{n}, y_{n+1}, t\right)=1$ for $t>0$, it follows that $\lim _{n \rightarrow \infty} F\left(y_{n}, y_{n+p}, t\right) \geq 1 \Delta 1 \Delta \cdots \Delta 1=1$, which shows that $\left\{y_{n}\right\}$ is a Cauchy sequence in $X$.

Now we come to our main result.

Theorem 2.2. Let $A, B, S$ and $T$ be four self-mappings of a Menger space $(X, F, \Delta)$ satisfying the conditions (2.1) and (2.2) and one of $A(X), B(X), S(X)$ and $T(X)$ is a complete subspace of $X$, then

1) $A$ and $S$ have a point of coincidence,

2) $B$ and $T$ have a point of coincidence.

Moreover, if the pairs $(A, S)$ and $(B, T)$ are weakly compatible, then $A, B, S$ and $T$ have a unique common fixed point.

Proof. Let $x_{0}$ be an arbitrary point in $X$. Then from (2.3), we have $y_{2 n}=T x_{2 n+1}=A x_{2 n}$ and $y_{2 n+1}=S x_{2 n+2}=$ $B x_{2 n+1}$. Then due to Lemma 2.1, $\left\{y_{n}\right\}$ is a Cauchy sequence in $X$. Now suppose that $S(X)$ is a complete subspace of $X$, then the subsequence $y_{2 n+1}=S x_{2 n+2}$ must get a limit in $S(X)$. Call it to be $u$ and $v \in S^{-1} u$. Then $S v=u$. As $\left\{y_{n}\right\}$ is a Cauchy sequence containing a convergent subsequence $\left\{y_{2 n+1}\right\}$, therefore the sequence $\left\{y_{n}\right\}$ also converges implying thereby the convergence of $\left\{y_{2 n}\right\}$ being a subsequence of the convergent sequence $\left\{y_{n}\right\}$.

On setting $x=v$ and $y=x_{2 n+1}$ in (2.2) one gets (for $t>$ $0)$,

$$
F\left(A v, y_{2 n+1}, t\right)=F\left(A v, B x_{2 n+1}, t\right) \geq \varphi\left(\min \left\{F\left(u, y_{2 n}, t\right), F(A v, u, t), F\left(y_{2 n+1}, y_{2 n}, t\right), F\left(u, y_{2 n+1}, 2 t\right), F\left(A v, y_{2 n}, t\right)\right\}\right)
$$

which on letting $n \rightarrow \infty$ reduces to $F(A v, u, t) \geq \varphi(F(A v, u, t))>F(A v, u, t)$ a contradiction. Therefore $A v=u=S v$, which shows that the pair $(A, S)$ has a point of coincidence. As $A(X) \subset T(X), A v=u$ implies that $u \in T(X)$. Let $w \in T^{-1} u$, then $T w=u$. On setting $x=x_{2 n}$ and $y=w$ in (2.2) one gets (for $t>0$ ),

$$
F\left(y_{2 n}, B w, t\right)=F\left(A x_{2 n}, B w, t\right) \geq \varphi\left(\min \left\{F\left(y_{2 n-1}, T w, t\right), F\left(y_{2 n-1}, y_{2 n}, t\right), F(u, B w, t), F\left(y_{2 n-1}, B w, 2 t\right), F\left(y_{2 n}, T w, t\right)\right\}\right)
$$

which on letting $n \rightarrow \infty$ reduces to $F(u, B w, t) \geq \varphi(F(u, B w, t))>F(u, B w, t)$, a contradiction. Therefore $u=B w$. Thus we have shown $u=A v=$ $S v=B w=T w$ which amounts to say that both pairs have point of coincidence. If one assumes $T(X)$ to be complete, then an analogous argument establishes this claim.

The remaining two cases pertain essentially to the previous cases. Indeed if $A(X)$ is complete, then $u \in A(X) \subset T(X)$ and if $B(X)$ is complete, then $u \in B(X) \subset S(X)$. Thus 1) and 2) are completely established. Since the pairs $(A, S)$ and $(B, T)$ are weakly compatible and $v$ and $w$ are their points of coincidence respectively, then $A u=A(S v)=S(A v)=S u$ and $B u=$ $B(T w)=T(B w)=T u$.

If $A u \neq u$, then on setting $x=u$ and $y=w$ in (2.2), one gets (for $t>0$ ), 


$$
\begin{aligned}
F(A u, u, t) & =F(A u, B w, t) \geq \varphi(\min \{(F(S u, T w, t), F(S u, A u, t), F(B w, T w, t), F(S u, B w, 2 t), F(A u, T w, t)\}) \\
& =\varphi(F(A u, u, t)>F(A u, u, t)
\end{aligned}
$$

a contradiction. Therefore $A u=u$. Similarly, one can show that $B u=u$. Thus $u$ is a common fixed point of $A$, $B, S$ and $T$. The uniqueness of a common fixed point follows easily. Also $u$ remains the unique common fixed point of both pairs separately. This completes the proof.

Example 2.2. Let $A, B, S$ and $T$ be self maps on Menger probabilistic metric space $X=[0,1]$ with usual metric $d$ defined by $d(x, y)=|x-y|$. For each $t \in(0, \infty)$, define $F(x, y, t)=\frac{t}{t+d(x, y)}$ and $F(x, y, 0)=0$, for all $x, y \in X$. Then $(X, F, \Delta)$ is a Menger probabilistic metric space, where $\Delta$ is defined by $\Delta(a, b)=\min (a, b)$. Define $A, B, S, T: X \rightarrow X$ by

$$
\begin{aligned}
& A x=\left\{\begin{array}{lll}
0 & \text { if } & x=0, \\
0.15 & \text { if } & x>0,
\end{array}\right. \\
& B x=\left\{\begin{array}{lll}
0 & \text { if } & x=0, \\
0.35 & \text { if } & x>0 ;
\end{array}\right. \\
& S x=\left\{\begin{array}{lll}
0 & \text { if } & x=0, \\
0.3 & \text { if } & 0<x \leq 0.5, \\
x-0.35 & \text { if } & x>0.5 ;
\end{array}\right. \\
& T x=\left\{\begin{array}{lll}
0 & \text { if } & x=0, \\
0.15 & \text { if } & 0<x \leq 0.5, \\
x-0.15 & \text { if } & x>0.5,
\end{array}\right.
\end{aligned}
$$

and define $\varphi:[0,1] \rightarrow[0,1]$ as $\varphi(0)=0, \varphi(1)=1$ and $\varphi(s)=\sqrt{s}$ for $0<s<1$. If we take $k=0.5$ and $t=1$, then we see that $A, B, S$ and $T$ satisfy all the conditions of the above theorem and have a unique common fixed point $0 \in X$. We note that the mappings $A$ and $S$ commute at the coincidence point $0 \in X$, and hence $A$ and $S$ are weakly compatible maps. Similarly $B$ and $T$ are weakly compatible maps. To see that the pairs $\{A, S\}$ and $\{B, T\}$ are non compatible, let us consider a decreasing sequence $\left\{x_{n}\right\}$ such that $x_{n} \rightarrow 0.5$. Then $A x_{n} \rightarrow 0.15, S x_{n} \rightarrow$ 0.15 , but

$$
\lim _{n \rightarrow \infty}\left(A S x_{n}, S A x_{n}, t\right)=\frac{t}{t+|0.15-0.3|} \neq 1 .
$$

Thus the pair $(A, S)$ is non compatible. Also $B x_{n} \rightarrow 0.35$, $T x_{n} \rightarrow 0.35$ but

$$
\lim _{n \rightarrow \infty}\left(B T x_{n}, T B x_{n}, t\right)=\frac{t}{t+|0.35-0.15|} \neq 1 .
$$

Hence, the pair $(B, T)$ is non compatible. All the mappings involved in this example are discontinuous at the common fixed point.

Theorem 2.3. Theorem 2.2 remains true if a "weakly compatible" property is replaced by any one (retaining the rest of the hypotheses) of the following:

1) $R$-weakly commuting property,

2) $R$-weakly commuting property of type 2 ),

3) $R$-weakly commuting property of type 1 ),

4) $R$-weakly commuting property of type 3 ),

5) weakly commuting property.

Proof. Since all the conditions of Theorem 2.2 are satisfied, then the existence of coincidence points for both the pairs is insured. Let $x$ be an arbitrary point of coincidence for the pair $(A, S)$, then using R-weak commutativity one gets $F(A S x, S A x, t) \geq F(A x, S x, t / R)=1$, which amounts to say that $A S x=S A x$. Thus the pair $(A, S)$ is coincidentally commuting. Similarly $(B, T)$ commutes at all of its coincidence points. Now applying Theorem 2.2, one concludes that $A, B, S$ and $T$ have a unique common fixed point. In case $(A, S)$ is an $R$-weakly commuting pair of type 2), then $F\left(A S x, S^{2} x, t\right) \geq F(A x, S x, t / R)=1$, which amounts to say that $A S x=S^{2} x$. Now,

$$
\begin{aligned}
& F(A S x, S A x, t) \geq F\left(A S x, S^{2} x, t / 2\right) \Delta F\left(S^{2} x, S A x, t / 2\right) \\
& =1 \Delta F\left(S^{2} x, S A x, t / 2\right)=F\left(S^{2} x, S A x, t / 2\right),
\end{aligned}
$$

which gives contradiction, yielding thereby $A S x=S A x$. Similarly, if pair is $R$-weakly commuting mappings of type 1$)$ or type 3$)$ or weakly commuting, then $(A, S)$ also commutes at their points of coincidence. Similarly, one can show that the pair $(B, T)$ is also weakly compatible. Now in view of Theorem 2.2, in all four cases $A, B, S$ and $T$ have a unique common fixed point. This completes the proof.

\section{Property (E.A.)}

Recently, Amari and Moutawakil [11] introduced a generalization of non compatible maps as property (E.A.).

Definition 3.1. Let $A$ and $S$ be two self-maps of a metric space $(X, d)$. The pair $(A, S)$ is said to satisfy property (E.A.), if there exists a sequence $\left\{x_{n}\right\}$ in $X$ such that $\lim _{n \rightarrow \infty} A x_{n}=\lim _{n \rightarrow \infty} S x_{n}=t$, for some $t \in X$.

Now in a similar mode we state property (E.A.) in Menger probabilistic metric spaces.

Definition 3.2. A pair of self-mappings $(f, g)$ of a Menger probabilistic metric space $(X, F, \Delta)$ is said to satisfy property(E.A.), if there exists a sequence $\left\{x_{n}\right\}$ in $X$ such that $\lim _{n \rightarrow \infty} F\left(f x_{n}, g x_{n}, t\right)=1$, for some $t \in X$.

Example 3.1 [11]. Let $X=[0,+\infty)$. Define 
$f, g: X \rightarrow X$ by $f x=\frac{x}{4}$ and $g x=\frac{3 x}{4}$, for all $x$ in $X$. Consider the sequence $x_{n}=\frac{1}{n}$. Cleary

$\lim _{n \rightarrow \infty} F\left(f x_{n}, g x_{n}, t\right)=1$. Then $f$ and $g$ satisfy property (E.A.).

Example 3.2 [11]. Let $X=[2,+\infty)$. Define

$f, g: X \rightarrow X$ by $g x=x+1$ and $f x=2 x+1$, for all $x \in$ $X$. Suppose that the property (E.A.) holds. Then, there exists a sequence $\left\{x_{n}\right\}$ in $X$ satisfying

$\lim _{n \rightarrow \infty} f x_{n}=\lim _{n \rightarrow \infty} g x_{n}=z$ for some $z \in X$. Therefore, $\lim _{n \rightarrow \infty} x_{n}=z-1$ and $\lim _{n \rightarrow \infty} x_{n}=\frac{z-1}{2}$. Thus, $z=1$, which is a contradiction, since 1 is not contained in $X$. Hence $f$ and $g$ do not satisfy property (E.A.).

Example 3.3 [12]. Let $(X, F, \Delta)$ be a Menger probabilistic metric space, where $X=[0,2]$ with minimum $t$ norm, and $F(x, y, t)=\frac{t}{t+d(x, y)}$ for all $t>0$ and for all $x, y \in X$. Define the self maps $f$ and $g$ as follows:

$$
f x=\left\{\begin{array}{lll}
2 & \text { if } & x \in[0,1], \\
\frac{x}{2} & \text { if } & 1<x \leq 2 ;
\end{array} \quad g x= \begin{cases}0 & \text { if } \quad x=1 \\
\frac{x+3}{5} & \text { otherwise }\end{cases}\right.
$$

Let $\left\{x_{n}=2-1 / n\right\}$ is a sequence in $X$ such that $\lim _{n \rightarrow \infty} f x_{n}=\lim _{n \rightarrow \infty} g x_{n}=z$. By definition of $f$ and $g$, we have $z \in\{1\}$. Thus $\{f, g\}$ satisfies property (E.A.). Two selfmappings $S$ and $T$ of a Menger space $(X, F, t)$ will be noncompatible if there exists at least one sequence $\left\{x_{n}\right\}$ in $X$ such that $\lim _{n \rightarrow \infty} S x_{n}=\lim _{n \rightarrow \infty} T x_{n}=z$ for some $z \in X$, but $\lim _{n \rightarrow \infty} F\left(S T x_{n}, T S x_{n}, t\right)$ is either not equal to 1 or non-existent. We note that noncompatible selfmappings of a Menger space $(X, F, \Delta)$ satisfy the property (E.A.).

Definition 3.3 [13]. Two pairs $(A, S)$ and $(B, T)$ of self mappings of a Menger PM space $(X, F, \Delta)$ are said to satisfy the common property E.A. if there exist two sequences $\left\{x_{n}\right\},\left\{y_{n}\right\}$ in $X$ and some $t$ in $X$ such that

$$
\begin{aligned}
\lim _{n \rightarrow \infty} A x_{n} & =\lim _{n \rightarrow \infty} S x_{n}=\lim _{n \rightarrow \infty} T y_{n} \\
& =\lim _{n \rightarrow \infty} B y_{n}=t
\end{aligned}
$$

Example 3.4 [13]. Let $(X, F, \Delta)$ be Menger space with $X=[-1,1]$ and

$$
F(x, y, t)=\left\{\begin{array}{lll}
e^{\frac{-|x-y|}{t}} & \text { if } t>0 \\
0 & \text { if } t=0
\end{array}\right.
$$

for all $x, y \in X$. Define self mappings $A, B, S$ and $T$ on $X$ as $A x=\frac{x}{3}, B x=-\frac{x}{3}, S x=x$ and $T x=-x$ for all $x \in$ $X$. Taking sequences $x_{n}=\frac{1}{n}$ and $y_{n}=-\frac{1}{n}$ in $X$, then $\lim _{n \rightarrow \infty} A x_{n}=\lim _{n \rightarrow \infty} S x_{n}=\lim _{n \rightarrow \infty} T y_{n}=\lim _{n \rightarrow \infty} B y_{n}=0$.
Thus, the pairs $(A, S)$ and $(B, T)$ share the common property (E.A.).

Now we prove the results of Kohli and Vashistha [1] for weakly compatible maps along with property (E.A.) as follows:

Theorem 3.1. Let $f$ and $g$ be weakly compatible self maps of a Menger probabilistic metric space $(X, F, \Delta)$ with continuous $t$-norm $\Delta$ satisfying $F(x, y, t)>0$ for all $x$, $y$ in $X$ and $t>0$ such that conditions (a-II) and (a-III) and the following holds:

(a-IV) $f$ and $g$ satisfy the property (E.A.),

(a-V) $g(X)$ is a closed subspace of $X$.

Then $f$ and $g$ have a unique common fixed point in $X$ provided $\mathrm{f}$ and $\mathrm{g}$ are weakly compatible maps.

Proof. Since $f$ and $g$ satisfy the property (E.A.) therefore, there exists a sequence $\left\{x_{n}\right\}$ in $X$ such that $\lim _{n \rightarrow \infty} f x_{n}=\lim _{n \rightarrow \infty} g x_{n}=u \in X$. As $g(X)$ is a closed subspace of $X$, therefore every convergent sequence of points of $g(X)$ has a limit point in $g(X)$. Therefore, $\lim _{n \rightarrow \infty} g x_{n}=\lim _{n \rightarrow \infty} f x_{n}=u=g a$, for some $a \in X$. This implies $u=g a \in g(X)$. Now we show that $f a=g a$. From (a-II), we have, $F\left(f a, f x_{n}, t\right) \geq r\left(F\left(g a, g x_{n}, t\right)\right)$. Proceeding limit as $n \rightarrow \infty$, we have,

$F(f a, u, t) \geq r(F(u, u, t))=r(1)=1$, this implies that $u$ $=g a=f a$. Thus $\mathrm{a}$ is the coincidence point of $f$ and $g$. Since $\mathrm{f}$ and $\mathrm{g}$ are weakly compatible, therefore, $f u=f g a$ $=g f a=g u$. Now we show that $f u=u$. From (a-II), we have, $F(f u, f a, q t) \geq r(F(g u, g a, t))$, which in turns implies that $f u=u$. Hence $\mathrm{u}$ is the unique common fixed point of $f$ and $g$. Uniqueness follows easily from (a-II).

Remark 3.1. It was pointed out in [14] that a pair of maps enjoying property (E.A.) relaxes the required containment of range of one mapping into the range of other which is utilized to construct the sequence of joint iterates. Moreover, it buys containment of ranges without any continuity requirements besides minimizes the commutativity conditions of the maps to the commutativity at their points of coincidence and it also allows replacing the completeness requirement of the space with a more natural condition of closeness of the range.

Example 3.5. [14] Consider $X=[-1,1]$ with the usual metric. Define the self-mappings $T$ and $I$ on $X$ as follows:

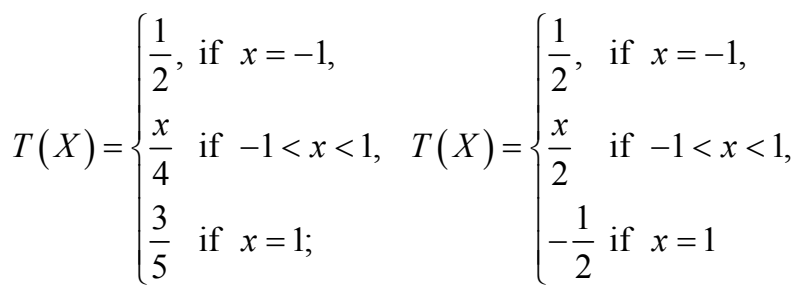

Consider the sequence $x_{n}=\frac{1}{n}$. Clearly,

$\lim _{n \rightarrow \infty} T x_{n}=\lim _{n \rightarrow \infty} I x_{n}=0$. Then $T$ and $I$ satisfy prop- 
erty (E.A.). Also, $T(X)=\left\{\frac{1}{2}, \frac{3}{5}\right\} \cup\left(-\frac{1}{4}, \frac{1}{4}\right)$ and $I(X)=\left[-\frac{1}{2}, \frac{1}{2}\right]$. Here one needs to note that neither $T(X)$ is contained in $I(X)$ nor $I(X)$ is contained in $T(X)$.

Now we prove existence of common fixed points for pairs of weakly compatible maps along with property (E. A.) using different types of control functions:

$(\Phi)$ : Consider the mapping $\phi:[0,1]^{5} \rightarrow[0,1]$, which is upper semi-continuous, nondecreasing in each co-ordinate variable and such that $\phi(1, t, 1,1, t) \geq t$, $\phi(1,1, t, t, 1) \geq t, \phi(t, 1,1, t, t) \geq t, \quad(t \in[0,1])$.

Theorem 3.2. Let $A, B, S$ and $T$ be self maps of a Menger probabilistic metric space $(X, F, \Delta)$ with continuous t-norm satisfying the following conditions:

1) $A(X) \subset T(X)$ and $B(X) \subset S(X)$,

2) $F(A x, B y, k t) \geq \phi(F(S x, T y, t), F(A x, S x, t)$,

$$
F(B y, T y, t), F(S x, B y, t), F(A x, T y, t)),
$$

for all $x, y$ in $X$ and $t>0$, where $k \in(0,1)$ and $\phi \in(\Phi)$,

3) pairs $(A, S)$ or $(B, T)$ satisfy property(E.A.),

4) pairs $(A, S)$ and $(B, T)$ are weakly compatible.

If the range of one of $A, B, S$ and $T$ is a closed subset of $X$, then $A, B, S$ and $T$ have a unique common fixed point in $X$.

Proof. Suppose that $(B, T)$ satisfies the property (E.A.). Then there exists a sequence $\left\{x_{n}\right\}$ in $X$ such that $\lim _{n \rightarrow \infty} B x_{n}=\lim _{n \rightarrow \infty} T x_{n}=z$ for some $z \in X$. Since $B(X) \subset S(X)$, therefore, there exists a sequence $\left\{y_{n}\right\}$ $\in X$ such that $\lim _{n \rightarrow \infty} B x_{n}=\lim _{n \rightarrow \infty} S y_{n}=z$. Hence

$\lim _{n \rightarrow \infty} S y_{n}=z$. Now we shall show that $\lim _{n \rightarrow \infty} A y_{n}=z$. We claim that $\lim _{n \rightarrow \infty} A y_{n}=l$.

For this we have from 2),

$$
\begin{aligned}
& F\left(A y_{n}, B x_{n}, k t\right) \geq \phi\left(F\left(S y_{n}, T x_{n}, t\right), F\left(A y_{n}, S y_{n}, t\right),\right. \\
& F\left(B x_{n}, T x_{n}, t\right), F\left(S y_{n}, B x_{n}, t\right), F\left(A y_{n}, T x_{n}, t\right)
\end{aligned}
$$

Proceeding limit as $n \rightarrow \infty$, we have, $F(l, z, k t) \geq \phi(1, F(l, z, t), 1,1, F(l, z, t)) \geq F(l, z, t)$, using $(\Phi)$ and by Lemma 1.1, we have $l=z$. Therefore, $\lim _{n \rightarrow \infty} A y_{n}=z$. Suppose that $S(X)$ is a closed subspace of $X$. Then $z=S u$ for some $u \in X$. Subsequently, we have

$$
\begin{aligned}
\lim _{n \rightarrow \infty} A y_{n} & =\lim _{n \rightarrow \infty} S y_{n}=\lim _{n \rightarrow \infty} T x_{n} \\
& =\lim _{n \rightarrow \infty} B x_{n}=z=S u .
\end{aligned}
$$

Now, we shall show that $\mathrm{Au}=\mathrm{z}$. From 2) we have,

$$
\begin{aligned}
& F\left(A u, B x_{n}, k t\right) \geq \phi\left(F\left(S u, T x_{n}, t\right), F(A u, S u, t),\right. \\
& \left.F\left(B x_{n}, T x_{n}, t\right), F\left(S u, B x_{n}, t\right), F\left(A u, T x_{n}, t\right)\right)
\end{aligned}
$$

Letting limit as $n \rightarrow \infty$,

$$
\begin{aligned}
F(A u, z, k t) & \geq \phi(1, F(A u, z, t), 1,1, F(A u, z, t)) \\
& \geq F(A u, z, t)
\end{aligned}
$$

using $(\Phi)$ and by Lemma 1.1, we have $A u=S u=z$. Since $A(X) \subset T(X)$, so there exists $v \in X$ such that $z$ $=A u=T v$. Now, we claim that $z=T v=B v$. Then From 2) we have,

$$
\begin{aligned}
& F(A u, B v, k t) \geq \phi(F(S u, T v, t), F(A u, S u, t), \\
& F(B v, T v, t), F(S u, B v, t), F(A u, T v, t))
\end{aligned}
$$

using $(\Phi)$ and by Lemma 1.1, we have $z=B v$. Thus we have $A u=S u=T v=B v=z$. Since the pair (A, S) is weak compatible which implies $A S u=S A u$, i.e, $A z=S z$.

Now we show that $A z=z$.

$$
\begin{aligned}
& F(A z, B v, k t) \geq \phi(F(S z, T v, t), F(A z, S z, t), \\
& F(B v, T v, t), F(S z, B v, t), F(A z, T v, t))
\end{aligned}
$$

Using $(\Phi)$ and by Lemma 1.1, $A z=S z=z$. The weak compatibility of $B$ and $T$ implies that $B T v=T B v$, i.e., $B z$ $=T z$. Now we shall show that $z$ is the common fixed point of $B$ and $T$. From 2), one obtain,

$$
\begin{aligned}
& F(A z, B z, k t) \geq \phi(F(S z, T z, t), F(A z, S z, t), \\
& F(B z, T z, t), F(S z, B z, t), F(A z, T z, t))
\end{aligned}
$$

using ( $\Phi)$ and by Lemma 1.1, $B z=z$. Hence $A z=B z=$ $S z=T z=z$ and $\mathrm{z}$ is a common fixed point of $A, B, S$ and $T$.

Example 3.6. Let $X=[0,2]$ equipped with the Euclidian distance and let $(X, F, \Delta)$ be the standard Menger probabilistic metric spaces induced by $(X, d)$, i.e., $F(x, y, t)=\frac{t}{t+d(x, y)}$. Define $A, B, S$ and $T$ by,

$$
\begin{aligned}
& A x=T x=\left\{\begin{array}{lll}
0 & \text { if } & 0 \leq x<1, \\
1 & \text { if } & 1 \leq x \leq 2
\end{array}\right. \\
& B x=S x=\left\{\begin{array}{lll}
0 & \text { if } & 0 \leq x<1, \\
\frac{1}{2} & \text { if } & 1 \leq x \leq 2 .
\end{array}\right.
\end{aligned}
$$

Let $\phi:[R+]^{5} \rightarrow[R+]$ be $\phi\left(x_{1}, x_{2}, x_{3}, x_{4}, x_{5}\right)=x_{1}$ and $k=1 / 2$ and consider $x_{n}=\frac{1}{n}$. Then,

$$
\begin{aligned}
\lim _{n \rightarrow \infty} A x_{n} & =\lim _{n \rightarrow \infty} S x_{n}=\lim _{n \rightarrow \infty} T x_{n} \\
& =\lim _{n \rightarrow \infty} B x_{n}=0 .
\end{aligned}
$$

Hence pairs $(A, S)$ and $(B, T)$ share property (E.A.). Also $A(X)=T(X)=\{0,1\}$ and $B(X)=S(X)=\{0,2\}$ are a closed subset of $X$. Moreover, pairs $(A, S)$ and $(B, T)$ are weakly compatible. Thus all the conditions of the above theorem are satisfied and 0 is the unique common fixed point of $A, B, S$ and $T$. 
In 2008, Kubiaczyk and Sharma [2] proved the following fixed point theorem.

Corollary 3.1 [2]. Let $A, B, S$ and $T$ be self maps of a Menger probabilistic metric space $(X, F, \Delta)$ with continuous t-norm satisfying 1), 3), 4) and the following:

5) $F(A x, B y, k t) \geq \min (F(S x, T y, t), F(A x, S x, t)$,

$$
F(B y, T y, t), F(S x, B y, t), F(A x, T y, t)),
$$

for all $x, y$ in $X$ and $t>0$, where $k \in(0,1)$. If the range of one of $A, B, S$ and $T$ is a closed subset of $X$, then $A, B$, $S$ and $T$ have a unique common fixed point in $X$.

Proof. Set $\phi\left(x_{1}, x_{2}, x_{3}, x_{4}, x_{5}\right)=\min \left(x_{1}, x_{2}, x_{3}, x_{4}, x_{5}\right)$ in Theorem 3.2.

Next we consider a function:

$(*) \psi:[0,1] \rightarrow[0,1]$ satisfying the conditions:

$\psi$ is continuous and nondecreasing on $[0,1]$ and $\psi(t)>t$ for all $t$ in $(0,1)$. We note that $\psi(1)=1$ and $\psi(t) \geq t$ for all $t$ in $[0,1]$, i.e., $\psi(F(x, y, t)) \geq F(x, y, t)$ holds for every $t>0$ and for all $x, y$ in $X$.

Theorem 3.3. Let $A, B, S$ and $T$ be self maps of a Menger probabilistic metric space $(X, F, \Delta)$ with continuous t-norm satisfying 1), 3), 4) and the following:

6) $F(A x, B y, t) \geq \psi(\min (F(S x, T y, t), F(A x, S x, t)$,

$$
F(B y, T y, t), F(S x, B y, t), F(A x, T y, t))),
$$

with $F(x, y, t)>0$ and $\psi \in(*)$ for all $x, y$ in $X$ and $t>0$. If the range of one of $A, B, S$ and $T$ is a closed subset of $X$, then $A, B, S$ and $T$ have a unique common fixed point in $X$.

Proof. Suppose that $(B, T)$ satisfies the property (E.A.). Then there exists a sequence $\left\{x_{n}\right\}$ in $X$ such that $\lim _{n \rightarrow \infty} B x_{n}=\lim _{n \rightarrow \infty} T x_{n}=z$ for some $z \in X$. Since, $B(X) \subset S(X)$, there exists a sequence $\left\{y_{n}\right\} \in X$ such that $\lim _{n \rightarrow \infty} B x_{n}=\lim _{n \rightarrow \infty} S y_{n}=z$. Hence

$\lim _{n \rightarrow \infty} S y_{n}=z$. We shall show that $\lim _{n \rightarrow \infty} A y_{n}=z$. From 6) we have,

$$
\begin{aligned}
& F\left(A y_{n}, B x_{n}, t\right) \geq \psi\left(\operatorname { m i n } \left(F\left(S y_{n}, T x_{n}, t\right), F\left(A y_{n}, S y_{n}, t\right),\right.\right. \\
& \left.\left.F\left(B x_{n}, T x_{n}, t\right), F\left(S y_{n}, B x_{n}, t\right), F\left(A y_{n}, T x_{n}, t\right)\right)\right)
\end{aligned}
$$

Proceeding limit as $n \rightarrow \infty$ and using $(*)$ one obtain, $\lim _{n \rightarrow \infty} A y_{n}=z$. Suppose that $S(X)$ is a closed subspace of $X$. Then $z=S u$ for some $u \in X$. Subsequently we have,

$$
\begin{aligned}
\lim _{n \rightarrow \infty} A y_{n} & =\lim _{n \rightarrow \infty} S y_{n}=\lim _{n \rightarrow \infty} T x_{n} \\
& =\lim _{n \rightarrow \infty} B x_{n}=z=S u .
\end{aligned}
$$

Now, we shall show that $A u=S u$. From 6) we have,

$$
\begin{aligned}
& F\left(A u, B x_{n}, t\right) \geq \psi\left(\operatorname { m i n } \left(F\left(S u, T x_{n}, t\right), F(A u, S u, t),\right.\right. \\
& \left.\left.F\left(B x_{n}, T x_{n}, t\right), F\left(S u, B x_{n}, t\right), F\left(A u, T x_{n}, t\right)\right)\right)
\end{aligned}
$$

Letting limit as $n \rightarrow \infty$, we get,

$$
\begin{aligned}
& F(A u, z, t) \geq \psi(\min (F(z, z, t), F(A u, z, t), \\
& F(z, z, t), F(z, z, t), F(A u, z, t)))
\end{aligned}
$$

using (*), we have, $A u=S u=z$. Since $A(X) \subset T(X)$, so there exists $v \in X$ such that $z=A u=T v$. Now, we claim that $\mathrm{z}=T v=B v$. From 6) we have,

$$
\begin{aligned}
& F(A u, B v, t) \geq \psi(\min (F(S u, T v, t), F(A u, S u, t), \\
& F(B v, T v, t), F(S u, B v, t), F(A u, T v, t)))
\end{aligned}
$$

using (*), we have, $z=B v$. Thus we have $A u=S u=T v$ $=B v=z$. Since the pair $(A, S)$ is weak compatible which implies $A S u=S A u$, i.e, $A z=S z$. From 6), we have,

$$
\begin{aligned}
& F(A z, B v, t) \geq \psi(\min (F(S z, T v, t), F(A z, S z, t), \\
& F(B v, T v, t), F(S z, B v, t), F(A z, T v, t)))
\end{aligned}
$$

using (*), we have, $A z=S z=z$. The weak compatibility of $\mathrm{B}$ and $\mathrm{T}$ implies that $B T v=T B v$, i.e., $B z=T z$. Now we shall show that $\mathrm{z}$ is the common fixed point of $A, B, T$ and $S$. Suppose that $B z \neq z$. Then using 6) one obtain,

$$
\begin{aligned}
& F(A z, B z, t) \geq \psi(\min (F(S z, T z, t), F(A z, S z, t), \\
& F(B z, T z, t), F(S z, B z, t), F(A z, T z, t)))
\end{aligned}
$$

using $(*)$, we have, $B z=z$. Hence $A z=B z=S z=T z=z$ and $z$ is a common fixed point of $A, B, S$ and $T$. Uniqueness follows easily.

Theorem 3.4. Let $A, B, S$ and $T$ be self maps of a Menger probabilistic metric space $(X, F, \Delta)$ with continuous t-norm satisfying 1), 2), 6) and the the following condition:

7) Pairs $(A, S)$ and $(B, T)$ satisfy a common property (E.A.).

If the range of $S$ and $T$ is a closed subset of $X$, then $A$, $B, S$ and $T$ have a unique common fixed point in $X$.

Proof. Suppose that $(A, S)$ and $(B, T)$ satisfy a common property(E.A.). Then there exists a sequences $\left\{x_{n}\right\}$ and $\left\{y_{n}\right\}$ in $X$ such that

$$
\begin{aligned}
\lim _{n \rightarrow \infty} A x_{n} & =\lim _{n \rightarrow \infty} S x_{n}=\lim _{n \rightarrow \infty} T y_{n} \\
& =\lim _{n \rightarrow \infty} B y_{n}=z
\end{aligned}
$$

for some $z \in X$. Since $S(X)$ and $T(X)$ are closed subsets of $X$, we obtain $z=S u=T v$ for some $u, v$ in $X$. From 6),

$$
\begin{aligned}
& F\left(A u, B y_{n}, t\right) \geq \psi\left(\operatorname { m i n } \left(F\left(S u, T y_{n}, t\right), F(A u, S u, t),\right.\right. \\
& \left.\left.F\left(B y_{n}, T y_{n}, t\right), F\left(S u, B y_{n}, t\right), F\left(A u, T y_{n}, t\right)\right)\right)
\end{aligned}
$$

Letting $n \rightarrow \infty$ and using $(*)$, we have, $z=A u=S u$ $=T v$.

The rest of the proof follows from the above theorem. 


\section{Occasionally Weakly Compatible Mappings}

In 2008, Al-Thagafi and Naseer Shahzad [15] introduced the concept of occasionally weakly compatible mappings.

Definition 4.1. Let $A$ and $T$ be selfmaps of a set $X$. If $A x=T x=w$ (say), $w \in X$, for some $x$ in $X$, then $x$ is called a coincidence point of $A$ and $T$ and the set of coincidence points of $A$ and $T$ in $X$ is denoted by $c(A, T)$, w is called a point of coincidence of $A$ and $T$.

Definition 4.2. Two self-maps $f$ and $g$ of a set $X$ are occasionally weakly compatible (shortly owc) iff there is a point $x$ in $X$ which is a coincidence point of $f$ and $g$ at which $f$ and $g$ commute.

Al-Thagafi and Naseer Shahzad [15] shown that occasionally weakly is weakly compatible but converse is not true.

Example 4.1. [15] Let $R$ be the usual metric space. Define $S, T: R \rightarrow R$ by $S x=2 x$ and $T x=x^{2}$ for all $x \in R$. Then $S x=T x$ for $x=0,2$ but $S T 0=T S 0$, and $S T 2 \neq T S 2$. $S$ and $T$ are occasionally weakly compatible self maps but not weakly compatible.

Remark 4.1. 1) Every pair of noncompatible selfmaps of a metric space $(X, d)$ satisfies property E.A., but its converse need not be true [16].

2) Weak compatibility and property E.A. are independent of each other [17].

3) Every compatible pair is weakly compatible but its converse need not be true [18].

4) Every weakly compatible pair is occasionally weakly compatible but its converse need not be true [19].

5) Occasionally weak compatibility and property E.A. are independent of each other [20].

Lemma 4.1 [21]. Let $X$ be a set and $f, g$ are owc self maps of $X$. If $f$ and $g$ have a unique point of coincidence, $w$ $=f x=g x$, then $\mathrm{w}$ is the unique common fixed point of $f$ and $g$.

Proposition 4.1. Let $A, B, S$ and $T$ be self maps of a Menger probabilistic metric space $(X, F, \Delta)$ with continuous t-norm satisfying (2.2) and the following conditions:

1) $B(X) \subseteq S(X)$, the pair $(B, T)$ satisfies property (E.A.) and $T(X)$ is a closed subspace of $X$; or

2) $A(X) \subseteq T(X)$, the pair $(A, S)$ satisfies property(E.A.) and $S(X)$ is a closed subspace of $X$, holds.

Then $c(A, S) \neq \phi$ and $c(B, T) \neq \phi$.

Proof: Suppose 1) holds.

Since the pair $(B, T)$ satisfies property (E.A.), then there exists a sequence $\left\{x_{n}\right\}$ in $X$ such that

$\lim _{n \rightarrow \infty} B x_{n}=\lim _{n \rightarrow \infty} T x_{n}=z$ for some $z \in X$. Since $B(X) \subset S(X)$, there exists a sequence $\left\{y_{n}\right\} \in X$ such that $\lim _{n \rightarrow \infty} B x_{n}=\lim _{n \rightarrow \infty} S y_{n}=z$. Now we claim that $\lim _{n \rightarrow \infty} A y_{n}=z$, for this purpose, suppose $\lim _{n \rightarrow \infty} A y_{n}=p$. Now put $x=y_{n}$, and $y=x_{n}$ in (2.2), we have,

$$
\begin{aligned}
& F\left(A y_{n}, B x_{n}, t\right) \geq \varphi\left(\operatorname { m i n } \left(F\left(S y_{n}, T x_{n}, t\right), F\left(A y_{n}, S y_{n}, t\right),\right.\right. \\
& \left.\left.F\left(B x_{n}, T x_{n}, t\right), F\left(S y_{n}, B x_{n}, 2 t\right), F\left(A y_{n}, T x_{n}, t\right)\right)\right)
\end{aligned}
$$

Letting $n \rightarrow \infty$, we have,

$$
F(p, z, t) \geq \varphi(1)=1 \text { imply that } p=z
$$

Case 1. $F(p, z, t) \geq \varphi(1)=1$ imply that $p=z$

Case 2. $F(p, z, t) \geq \varphi(F(p, z, t)>F(p, z, t)$, a contradiction. Hence, $p=z$, i.e., $\lim _{n \rightarrow \infty} A y_{n}=z$. Since $T(X)$ is a closed subspace of $X$, therefore $z \in T(X)$ and this implies $z=T v$ for some $v \in X$. If $B v \neq z$, then on putting $x=y_{n}$ and $y=v$ in (2.2), we have,

$$
\begin{aligned}
& F\left(A y_{n}, B v, t\right) \geq \varphi\left(\operatorname { m i n } \left(F\left(S y_{n}, T v, t\right), F\left(A y_{n}, S y_{n}, t\right),\right.\right. \\
& \left.\left.F(B v, T v, t), F\left(S y_{n}, B v, 2 t\right), F\left(A y_{n}, T v, t\right)\right)\right)
\end{aligned}
$$

Letting $n \rightarrow \infty$, we have,

$$
\begin{aligned}
& F(z, B v, t) \geq \varphi(\min (1,1, F(B v, z, t), F(z, B v, 2 t), 1)) \\
& \geq \varphi(\min (1,1, F(B v, z, t), \Delta(1, F(z, B v, 2 t)), 1))
\end{aligned}
$$

Case 1. $F(z, B v, t) \geq \varphi(1)=1$ imply $B v=z$

Case 2. $F(z, B v, t) \geq \varphi(F(z, B v, t)>F(z, B v, t)$, a contradiction. Hence, $B v=z=T v$, therefore $c(B, T) \neq \phi$.

Now, since $B(X) \subseteq S(X)$ and $z \in B(X)$, there exists a $u \in X \quad$ such that $z=S u$.

If, $A u \neq z$, then on putting $x=u$ and $y=v$ in (2.2), we have,

$F(A u, B v, t) \geq \varphi(\min (F(S u, T v, t), F(A u, S u, t)$,

$F(B v, T v, t), F(S u, B v, 2 t), F(A u, T v, t)))$

i.e., $F(A u, z, t) \geq \varphi(\min (1, F(A u, z, t), 1,1, F(A u, z, t)))$

Case 1. $F(A u, z, t) \geq \varphi(1)=1$ imply $A u=z$

Case 2. $F(A u, z, t) \geq \varphi(F(A u, z, t))>F(A u, z, t)$, a contradiction. Hence, $A u=z=S u$.

Thus $c(A, S) \neq \phi$.

Similarly, the assertion holds under assumption 2).

Hence, Proposition 4.1 follows.

Theorem 4.1. In addition to hypothesis of Proposition 4.1 on $A, B, S$ and $T$, if both the pairs $(A, S)$ and $(B, T)$ are owc on $X$, then the maps $A, B, S$ and $T$ have a unique common fixed point in $X$.

Proof: By Proposition 4.1, $c(A, S) \neq \phi$, and $c(B, T) \neq \phi$. Since the pair $(A, S)$ is owc, therefore there exists $u_{1} \in c(A, S)$ such that $A u_{1}=S u_{1}=z_{1}$ (say) and $A S u_{1}=S A u_{1}$, therefore $A z_{1}=S z_{1}=z_{2}$ (say). Since the pair $(B, T)$ is owc, therefore there exists $v_{1} \in c(B, T)$ such 
that $B v_{1}=T v_{1}=w$ (say) and $B T v_{1}=T B v_{1}$, i.e., $B w=T w=$ $w_{1}$ (say). Next we claim that $z_{2}=w_{1}$. If $z_{2} \neq w_{1}$, then form (2.2), we have,

$$
\begin{aligned}
& F\left(z_{2}, w_{1}, t\right)=F\left(A z_{1}, B w, t\right) \\
& \geq \varphi\left(\operatorname { m i n } \left(F\left(S z_{1}, T w, t\right), F\left(A z_{1}, S z_{1}, t\right), F(B w, T w, t),\right.\right. \\
& \left.\left.\quad F\left(S z_{1}, B w, 2 t\right), F\left(A z_{1}, T w, t\right)\right)\right)
\end{aligned}
$$

imply $z_{2}=w_{1}$. Therefore, we have $A z_{1}=S z_{1}=w_{1}$. Next we show that $z_{1}=w_{1}$, form (2.2), we have,

$$
\begin{aligned}
& F\left(z_{1}, w_{1}, t\right)=F\left(A z_{1}, B w, t\right) \\
\geq & \varphi\left(\operatorname { m i n } \left(F\left(S u_{1}, T w, t\right), F\left(A u_{1}, S u_{1}, t\right), F(B w, T w, t),\right.\right. \\
& \left.\left.F\left(S u_{1}, B w, 2 t\right), F\left(A u_{1}, T w, t\right)\right)\right)
\end{aligned}
$$

imply that $w_{1}=z_{1}$. Thus $A z_{1}=S z_{1}=z_{1}$ and $B w=T w=z_{1}$. Next we claim that $w=z_{1}$. If $w \neq z_{1}$, then from (2.2), we have,

$$
\begin{aligned}
& F\left(z_{1}, w, t\right)=F\left(A z_{1}, B v_{1}, t\right) \\
& \geq \varphi\left(\operatorname { m i n } \left(F\left(S z_{1}, T v_{1}, t\right), F\left(A z_{1}, S z_{1}, t\right), F\left(B v_{1}, T v_{1}, t\right),\right.\right. \\
& \left.\left.\quad F\left(S z_{1}, B v_{1}, 2 t\right), F\left(A z_{1}, T v_{1}, t\right)\right)\right)
\end{aligned}
$$

Imply $w=z_{1}$. Hence we have $B z_{1}=T z_{1}=z_{1}$. Therefore, we obtain $A z_{1}=T z_{1}=B z_{1}=S z_{1}=z_{1}$.

Uniqueness follows easily.

Example 4.2. Let $X=[0,2]$ equipped with the Euclidian distance and the Menger spaces induced by $(X, d)$, i.e., $F(x, y, t)=\frac{t}{t+d(x, y)}$. Clearly $(X, F, \Delta)$ is a Menger space with $\Delta(a, b)=\min \{a, b\}$. Define the self maps $A$, $B, S$ and $T$ on $X$ by

$$
\begin{aligned}
& A x=\left\{\begin{array}{ll}
0 & \text { if } x=0, \\
0.15 & \text { if } x>0 ;
\end{array} \quad B x= \begin{cases}0 & \text { if } x=0, \\
0.35 & \text { if } x>0 ;\end{cases} \right. \\
& S x= \begin{cases}0 & \text { if } x=0, \\
0.40 & \text { if } 0<x \leq 0.6, \\
x-0.45 & \text { if } x>0.6 ;\end{cases} \\
& T x= \begin{cases}0 & \text { if } x=0, \\
0.25 & \text { if } 0<x \leq 0.6, \\
x-0.25 & \text { if } x>0.6\end{cases}
\end{aligned}
$$

Now

$$
\begin{aligned}
& A(X)=\{0,0.15\}, B(X)=\{0,0.35\}, \\
& S(X)=\{0\} \cup(0.15,1.55), \\
& T(X)=\{0\} \cup\{0.25\} \cup(0.35,1.75)
\end{aligned}
$$

and taking $\varphi(t)=\sqrt{t}$ for $0<t<1$. We observe that $B(X) \subset S(X), T(X)$ is a closed subset of $X$, and neither $A(X) \subseteq T(X)$ nor $T(X) \subseteq A(X)$. The selfmaps $A, B$,
$S$ and $T$ satisfy the inequality (2.2). Let us consider the sequence $x_{n}=0.60+\frac{1}{n}, n=1,2,3 \cdots$ Then $A x_{n} \rightarrow 0.15$, $B x_{n} \rightarrow 0.35, S x_{n} \rightarrow 0.15, \quad T x_{n} \rightarrow 0.35, \quad A S x_{n} \rightarrow 0.15$, $S A x_{n} \rightarrow 0.40, \quad B T x_{n} \rightarrow 0.35, \quad T B x_{n} \rightarrow 0.25$.

One can see that $\lim _{n \rightarrow \infty} B x_{n}=\lim _{n \rightarrow \infty} T x_{n}=0.35$, so that the pair $(B, T)$ satisfies property (E.A.). But the pair $(B, T)$ is not compatible for $\lim _{n \rightarrow \infty} F\left(B T x_{n}, T B x_{n}, t\right) \neq 1$. Clearly, the pairs $(A, S)$ and $(B, T)$ are owc. Hence, the selfmaps $A, B, S$, and $T$ satisfy all the conditions of Theorem 4.1 and 0 the unique common fixed point of $A$, $B, S$ and $T$. Moreover, $A, B, S$ and $T$ are discontinuous at the fixed point 0 .

Theorem 4.2. Let $(X, F, \Delta)$ be a complete Menger space and let $A, B, S$ and $T$ be self-mappings of $X$. Let the pairs $(A, S)$ and $(B, T)$ be owc and for $k \in(0,1)$ such that

$$
\text { 3) } \begin{aligned}
& F(A x, B y, k t) \geq \min (F(S x, T y, t), F(A x, S x, t), \\
& F(B y, T y, t), F(S x, B y, 2 t), F(A x, T y, t)),
\end{aligned}
$$

for all $x, y \in X$ and for all $t>0$, then there exists a unique point $w \in X$ such that $A w=S w=w$ and a unique point $z \in X$ such that $B z=T z=z$. Moreover, $z$ $=w$, so that there is a unique common fixed point of $A, B$, $S$ and $T$.

Proof: Since the pairs $(A, S)$ and $(B, T)$ are owc, so there exists $x, y \in X$ such that $A x=S x$ and $B y=T y$. We claim that $A x=B y$. If $A x \neq B y$, then by 3),

$$
\begin{aligned}
& F(A x, B y, k t) \geq \min (F(S x, T y, t), F(A x, S x, t), \\
& F(B y, T y, t), F(S x, B y, 2 t), F(A x, T y, t)), \\
\geq & \min \{F(S x, T y, t), F(S x, A x, t), F(B y, T y, t), \\
& \Delta(F(S x, A x, t) F(A x, B y, t)), F(A x, T y, t)\} \\
= & F(A x, B y, t) .
\end{aligned}
$$

Then by Lemma 1.1, we have, $A x=B y$, i.e., $A x=S x=$ $B y=T y$. Suppose that there is another point $\mathrm{z}$ such that $A z=S z$, then by 3) $A z=S z=B y=T y$. So, $A x=A z$ and $w=A x=S x$ is the unique point of coincidence of $A$ and $S$. By Lemma 4.1, $w$ is the only common fixed point of $A$ and $S$. Similarly, there is a unique point $z \in X$ such that $z$ $=B z=T z$. Assume that $w \neq z$, then by 3 ), we have,

$$
\begin{aligned}
& F(w, z, k t)=F(A w, B z, k t) \\
\geq & \min (F(S w, T z, t)) F(A w, S w, t), F(B z, T z, t), \\
& F(S w, B z, 2 t), F((A w, T z, t)) \\
\geq & \min \{F(S w, T z, t), F(S w, A w, t), F(B z, T z, t), \\
& \Delta(F(S w, A w, t), F(A w, B z, t)), F((A w, T z, t)\} \\
= & F(A w, B z, t)=F(w, z, t) .
\end{aligned}
$$


In view of Lemma 1.1, we have $z=w$ and $z$ is a common fixed point of $A, B, S$ and $T$.

Uniqueness follows easily from 3 ).

Theorem 4.3. Let $(X, F, \Delta)$ be a complete Menger space and let $A, B, S$ and $T$ be self-mappings of $X$. Let the pairs $(A, S)$ and $(B, T)$ be owc and for $k \in(0,1)$ such that

4) $F(A x, B y, k t) \geq \phi(F(S x, T y, t), F(A x, S x, t)$,

$$
F(B y, T y, t), F(S x, B y, 2 t), F(A x, T y, t)),
$$

for all $x, y \in X$ and $\phi:[0,1]^{5} \rightarrow[0,1]$ such that $\phi(t, 1,1, t, t)>t$ for all $0<t<1$, then there exists a unique common fixed point of $A, B, S$ and $T$.

Proof: Since the pairs $(A, S)$ and $(B, T)$ are owc, there are points $x, y \in X$ such that $A x=S x$ and $B y=T y$. We claim that $A x=B y$. If not then by 4)

$$
\begin{aligned}
& F(A x, B y, k t) \geq \phi(F(S x, T y, t), F(A x, S x, t), \\
& F(B y, T y, t), F(S x, B y, 2 t), F(A x, T y, t)), \\
& \geq \phi\{F(S x, T y, t), F(S x, A x, t), F(B y, T y, t), \\
& \Delta(F(S x, A x, t), F(A x, B y, t)), F(A x, T y, t)\} \\
& \geq \phi\{F(A x, B y, t), 1,1, \Delta(1, F(A x, B y, t)), F(A x, B y, t)\} \\
& >F(A x, B y, t),
\end{aligned}
$$

a contradiction, therefore $A x=B y$, i.e., $A x=S x=B y=$ $T y$ and rest of the proof follows from Theorem 4.2 by replacing inequality 3 ) with 4 ).

Corollary 4.1. Let $(X, F, \Delta)$ be a complete Menger space and let $\mathrm{A}, \mathrm{B}, \mathrm{S}$ and $\mathrm{T}$ be self-mappings of $X$. Let the pairs $(A, S)$ and $(B, T)$ be owc. If there exists a point $k \in(0,1)$ for all $x, y \in X$ and $t>0$, such that

5) $F(A x, B y, k t) \geq F(S x, T y, t)$,

then there exists a unique common fixed point of $A, B, S$ and $T$.

Proof: The proof follows from Theorem 4.2.

Theorem 4.4. Let $(X, F, \Delta)$ be a complete Menger space. Then continuous self mappings $S$ and $T$ of $X$ have a common fixed point in $X$ if and only if there exists a self mapping $A$ of $X$ such that the following conditions are satisfied.

6) $A(X) \subset T(X) \cap S(X)$,

7) The pairs $(A, S)$ and $(A, T)$ are weakly compatible,

8) There exists a point $k \in(0,1)$ for all $x, y \in X$ and $t>0$, such that

$$
\begin{aligned}
& F(A x, A y, k t) \geq F(S x M T y, t) \Delta F(A x, S x, t) \\
& \Delta F(A y, T y, t) \Delta F(A x, T y, t)
\end{aligned}
$$

Then $A, S$ and $T$ have a unique common fixed point.

Proof: Since weakly compatible implies owc, the result follows from Theorem 4.2.

Theorem 4.5. Let $(X, F, \Delta)$ be a complete Menger space and let $A$ and $S$ be self-mappings of $X$. Let the pair $(A, S)$ be owc. If there exists a point $k \in(0,1)$ for all $x, y \in X$ and $t>0$, such that

$$
\text { 9) } \begin{aligned}
& F(S x, S y, k t) \geq a F(A x, A y, t) \\
+ & b \min \{(F(A x, A y, t), F(S x, A x, t), F(S y, A y, t)\}
\end{aligned}
$$

where $a, b>0, a+b>1$. Then $\mathrm{A}$ and $\mathrm{S}$ have a unique common fixed point.

Proof: Since the pair $(A, S)$ is owc, so there is a point $x \in X$ such that $A x=S x$. Suppose that there exist another point $y \in X$ for which $A y=S y$. We claim that $S x$ $=S y$. By inequality 9) we have,

$$
\begin{aligned}
F & (S x, S y, k t) \geq a F(A x, A y, t) \\
& +b \min \{(F(A x, A y, t), F(S x, A x, t), F(S y, A y, t)\} \\
= & a F(S x, S y, t)+b \min \{F(S x, S y, t), \\
& F(S x, S x, t), F(S y, S y, t)\} \\
= & (a+b) F(S x, S y, t),
\end{aligned}
$$

a contradiction, since $(a+b)>1$. Therefore $S x=S y$. Therefore $A x=A y$ and $A x$ is unique.

From Lemma 4.1, $A$ and $S$ have a unique fixed point.

\section{Acknowledgements}

First author is highly thankful to University Grant Commission, New Delhi-11016, INDIA for providing Major Research Project under F. No. 39-41|2010(SR).

\section{REFERENCES}

[1] J. K. Kohli and S. Vashistha, "Common Fixed Point Theorems in Probabilistic Metric Spaces," Acta Mathematica Hungarica, Vol. 115, No. 1-2, 2007, pp. 37-47. doi:10.1007/s10474-006-0533-7

[2] I. Kubiaczyk and S. Sharma, "Some Common Fixed Point Theorems in Menger Space under Strict Contractive Conditions," Southeast Asian Bulletin of Mathematics, Vol. 32, No. 1, 2008, pp. 117-124.

[3] K. Menger, "Statistical Metrices," Proceedings of the National Academy of Sciences USA, Vol. 28, No. 12, 1942, pp. 535-537. doi:10.1073/pnas.28.12.535

[4] V. M. Sehgal and A. T. Bharucha-Reid, "Fixed Points of Contraction Mappings on Probabilistic Metric Spaces," Mathematical Systems Theory, Vol. 6, No. 1, 1972, pp. 97-102. doi:10.1007/BF01706080

[5] B. Schweizer and A. Sklar, "Probabilistic Metric Spaces," North Holland, Amsterdam, 1983.

[6] S. Sessa, "On a Weak Commutativity Condition of Mappings in Fixed Point Considerations," Publications DeL'Institut Mathematique, Nouvelle Serie Tome 32, 1982, pp. 149-153.

[7] G. Jungck, "Compatible Mappings and Common fixed 
Points," International Journal of Mathematics and Mathematical Sciences, Vol. 9, No. 4, 1986, pp. 771-779. doi:10.1155/S0161171286000935

[8] S. N. Mishra, "Common Fixed Points of Compatible Mappings in PM-Spaces," Mathematica Japonica, Vol. 36, No. 2, 1991, pp. 283-289.

[9] G. Jungck, "Common Fixed Points for Non-Continuous Non-Self Maps on Non-Metric Spaces," Far East Journal of Mathematical Sciences, Vol. 4, No. 2, 1996, pp. 199215.

[10] D. Mihet, "A Note on a Common Fixed Point Theorem in Probabilistic Metric Spaces," Acta Mathematica Hungarica, Vol. 125, No. 1-2, 2009, pp. 127-130. doi:10.1007/s10474-009-8238-3

[11] M. Aamri and D. El Moutawakil, "Some New Common fixed Point Theorems under Strict Contractive Conditions," Journal of Mathematical Analysis and Application, Vol. 27, 2002, pp. 181-188. doi:10.1016/S0022-247X(02)00059-8

[12] M. Abbas, I. Altun and D. Gopal, "Common Fixed Point Theorems for Non Compatible Mappings in Fuzzy Metric Spaces," Bulletin of Mathematical Analysis and Applications, Vol. 1, No. 2, 2009, pp. 47-56.

[13] M. Imdad, M. Tanveer and M. Hasan, "Some Common Fixed Point Theorems in Menger PM Spaces," 2011, in Press.

[14] M. I. J. Ali, "Jungck's Common Fixed Point Theorem and E.A. Property," Acta Mathematica Sinica, Vol. 24, No. 1,
2008, pp. 87-94. doi:10.1007/s10114-007-0990-0

[15] M. A. Al-Thagafi and N. Shahzad, "Generalized I-Nonexpansive Selfmaps and Invariant Approximations," Acta Mathematica Sinica, English Series, Vol. 24, No. 5, 2008, pp. 867-876. doi:10.1007/s10114-007-5598-x

[16] G. V. R. Babu and G. N. Alenmayehu, "A Common Fixed Point Theorem for Weakly Contractive Mappings Satisfying Property (E.A.)," Applied Mathematics ENotes, Vol. 10, 2010, pp. 167-174.

[17] H. K. Pathak, R. Rodriguez-Lopez and R. K. Verma, "A Common Fixed Point Theorem Using Implicit Relation and Property (E.A.) in Metric Spaces," Filomat, Vol. 21, No. 2, 2007, pp. 211-234. doi:10.2298/FIL0702211P

[18] G. Jungck and B. E. Rhoades, "Fixed Points for Set- Valued Functions without Continuity," Indian Journal of Pure and Applied Mathematics, Vol. 29, No. 3, 1998, pp. 227-238.

[19] M. A. Al-Thagafi and N. Shahzad, "A Note on Occasionally Weakly Compatible Maps," International Journal of Mathematical Analysis, Vol. 3, No. 2, 2009, pp. 55-58.

[20] G. V. R. Babu and G. N. Alenmayehu, "Points of Coincidence and Common Fixed Points of a Pair of Generalized Weakly Contractive Mappings," Journal of Advanced Research in Pure Mathematics, Vol. 2, No. 2, 2010, pp. 89-106. doi:10.5373/jarpm.338.010810

[21] G. Jungck and B. E. Rhoades, "Fixed Point Theorems for Occasionally Weakly Compatible Mappings," Fixed Point Theory, Vol. 7, 2006, pp. 286-296. 